\title{
Size dependence of electrical conductivity and thermo- electric enhancements in spin-coated PEDOT:PSS single and multiple layers
}

\author{
Virgil Andrei, ${ }^{a, \ddagger}$ Kevin Bethke,${ }^{a}$ Fani Madzharova, ${ }^{a}$ Sebastian Beeg, ${ }^{b, c}$ Axel Knop-Gericke, ${ }^{b}$ \\ Janina Kneipp, ${ }^{a}$ and Klaus Rademann*a
}

\begin{abstract}
Despite the recent progress in the field of conductive polymers, a deeper understanding is still required to explain the differences between reported data. In this work, we reveal that the electrical conductivity $\sigma$ of a poly(3,4-ethylenedioxythiophene)-poly(styrenesulfonate) (PEDOT:PSS) film can be significantly increased by spin-coating multiple thin layers onto a substrate. Generally, $\sigma$ can be improved by more than fourfold for multiple layers, as compared to a single thicker one. A gradual enhancement is observed for pristine PEDOT:PSS films (up to $2.10 \pm 0.26 \mathrm{~S} \mathrm{~cm}^{-1}$ for 5 layered films), while a plateau in $\sigma$ at around $200 \mathrm{~S} \mathrm{~cm}^{-1}$ is reached after only three layers, when using a PEDOT:PSS solution with $5 \mathrm{vol} . \%$ dimethyl sulfoxide (DMSO). In contrast, only a small change in $\sigma$ is observed for single layers of varying thickness. Accordingly, the thermoelectric power factor is also increased by up to 3.4 times for the multiple layers. Based on AFM, XPS, UV-Vis and Raman spectroscopy measurements, two mechanisms are also proposed, involving an increase in percolation by inclusion of smaller grains within the existing ones, respectively a reorganization of the PEDOT:PSS chains. Firstly, these findings represent a direct strategy for enhancing the thermoelectric performance of conductive polymer films without additional reagents. Secondly, the mechanistic insights contribute to understand the broader picture, by explaining existing literature results.
\end{abstract}

\section{Introduction}

Since their introduction by Heeger et al., conductive polymers have been employed in a broad range of applications and fields, from electronic circuits and sensors, to light diodes, solar cells and flexible electrodes. $\frac{1}{\underline{1}}$ To enable these advances, the outstanding molecular adaptability of the conjugated chains has been put to good use, moving beyond the simple polyacetylene, to complex

\footnotetext{
0 a Department of Chemistry, Humboldt-Universität zu Berlin, Brook-Taylor-Straße 2, 12489 Berlin, Germany. Fax: +49 30/2093-5559; Tel: +49 30/2093-5565; E-mail: klaus.rademann@chemie.hu-berlin.de

$0 b$ Department of Inorganic Chemistry, Fritz-HaberInstitut der Max-Planck-Gesellschaft, Faradayweg 4-6, 14195 Berlin, Germany.

$0 c$ Department of Heterogeneous Reactions, Max-PlanckInstitute for Chemical Energy Conversion, Stiftstraße 34-36, 45470 Mülheim an der Ruhr, Germany.

$0_{\ddagger}{ }^{\circ}$ Current address: Department of Chemistry, University of Cambridge, Lensfield Road, Cambridge CB2 1EW, UK.
}

structures such as poly(3,4-ethylenedioxythiophene)poly(styrenesulfonate) (PEDOT:PSS), $\underline{2-5}$ poly(3hexylthiophene) (P3HT), $\underline{6,7}$ or protonated polyaniline (PANI) salts. $\stackrel{-11}{-11}$ During the last decade, these conjugated polymers and their composites have also received a significant interest from the thermoelectric (TE) community, $\stackrel{2,12}{=}$ due to their high electrical conductivity $(\sigma)$ and tunable Seebeck coefficient (or thermopower, $\mathrm{S})$, which result in power factors $(\mathrm{PF}=$ $S^{2} \sigma$ ) comparable to those of carbon based compounds, $13-16$ transition metal oxides, $17-21$ or even the established tellurides. ${ }^{22,23}$ Moreover, their remarkably low thermal conductivity $\kappa$ contributes to a high figure of merit $\left(\mathrm{ZT}=S^{2} \sigma T / \kappa\right)$, which is an established indicator for the thermoelectric energy conversion efficiency. 12

Within this class of materials, PEDOT:PSS currently stands out as one of the most promising TE compounds, with $\sigma$ between $0.007-4600 \mathrm{~S} \mathrm{~cm}^{-1}$ and $\mathrm{S}$ typically between $7 \mu \mathrm{V} \mathrm{K}^{-1}$ to $55 \mu \mathrm{V} \mathrm{K}^{-1}$, de- 
pending on factors such as vendor, $\underline{2}$ type of solution, $\stackrel{\underline{2}}{=}$ sample preparation,,$\underline{3,24}-\underline{29}$ annealing conditions $30-33$ and dopants. $1,2,4,34$ While the electrical conductivities of commercially available products are often below $1000 \mathrm{~S} \mathrm{~cm}^{-1}, \underline{\underline{4}}$ further enhancements have been obtained by either methanesulfonic acid treatment $\left(3300 \mathrm{~S} \mathrm{~cm}^{-1}\right), \frac{32}{, 2}$ concentrated sulfuric acid treatment $\left(4000 \mathrm{~S} \mathrm{~cm}^{-1}\right), \underline{33}$ or solution shearing $\left(4600 \mathrm{~S} \mathrm{~cm}^{-1}\right) .28$ A similar effect can be also observed for the Seebeck coefficient, which can be significantly enhanced to values surpassing $400 \mu \mathrm{VK}^{-1}, \underline{35}$ by tuning the positive charging of the PEDOT:PSS chains (i.e. the doping level) under the influence of reducing agents $\frac{36}{6}$ or electrochemically. $\cdot 35$

Even if significant steps have been made towards elucidating the conduction mechanisms, $\underline{1,37-39}$ a deeper understanding is still required to explain these large deviations occurring in the reported literature. Although quantum-effects, such as quantumconfinement and boundary scattering, $\underline{40,41}$ can affect the transport properties at the nanoscale, the influence of the film thickness on the electrical transport is yet to be systematically investigated. While the results presented in a few recent reports give some hints towards a higher performance in thicker conductive layers, $, 5,23,26$ these enhancements are rather attributed to the surface treatment, 26 or the physical interactions between different components (e.g. coiling of PANI chains along carbon nanotubes). $\underline{\underline{22,23}}$ In order to clarify this, two main strategies must be taken into account, namely the adjustment of film thickness by decreasing the spin-coating speed, or by deposition of several layers. In this work, we reveal the differences between the electrical conductivities in both cases, proposing a mechanistic explanation based on extensive experimental data. Moreover, these results are discussed from a thermoelectric point of view, with a focus on the performance enhancement for practical applications.

\section{Methods}

To obtain a $1 \mathrm{wt} . \%$ polymeric solution, $200 \mathrm{mg}$ of PEDOT:PSS (Orgacon ${ }^{T M}$ DRY, product of AgfaGevaert, purchased from Sigma-Aldrich) is first dis- solved in $19.8 \mathrm{~mL}$ distilled water and stirred at room temperature, at a speed of 1000 revolutions per minute (RPM). The stirring occurs for 48 hours, to ensure the homogeneity of the polymer suspension. The conductive PEDOT:PSS layers are spincoated on top of $\approx 25 \times 25 \mathrm{~mm}^{2}$ glass plates, which are made hydrophilic by submerging in a Piranha solution for $20 \mathrm{~min}$. The Piranha solution consists of freshly mixed sulfuric acid (Fluka, 95-97\%) and hydrogen peroxide (Sigma-Aldrich, 30\%), in a volume ratio of 7:3. After the spin-coating of each layer, the glass plates are kept in a drying oven at $403 \mathrm{~K}$ for $5 \mathrm{~min}$, to remove the remaining solvent. Two parallel conductive stripes are applied through a paper mask using Ferro GmbH silver paste, to improve the electrical contact. The distance between both stripes amounts to $17 \mathrm{~mm}$.

A Chemat Technology KW-4A Spin-Coater is employed for obtaining the polymer layers. The spincoating process requires the prior setting of two pairs of parameters: $\mathrm{t} 1, \mathrm{v} 1$ (the time and rotation speed at which the spreading occurs), respectively t2, v2 (the time and spinning speed for removing the excess liquid by centrifugation). Both t1 and t2 are kept constant at $15 \mathrm{~s}$, respectively $60 \mathrm{~s}$ during the investigations. While $\mathrm{v} 1$ is maintained at a constant value of $500 \mathrm{RPM}, \mathrm{v} 2$ is varied between 1000-3000 RPM for the PEDOT:PSS films.

The Seebeck coefficient is determined using a custom-made setup, which consists of a proportional-integral-derivative (PID) controller, two Peltier elements and a Fluke 289 multimeter (see Fig. (1). A similar setup employs a VersaSTAT3 current source to measure the quasi-4-point electrical resistance at room temperature (Fig. S4, Supporting Information). The thickness of the layers $(h)$ is determined with a Nanosurf ${ }^{\circledR}$ Mobile $\mathrm{S}$ atomic force microscope (AFM) operating in a Dynamic Force mode (scan rate of 1 line per second, with 128 points per line), which records the thickness of three scratches along the sample length (Fig. S7, Supporting Information). The software Nanosurf Mobile S 2.2 records the AFM images, while the program Gwyddion 2.42 is used to read and interpret the raw data.

Ultraviolet-visible light (UV-Vis) spectra are recorded with a Jasco V-670 UV-Vis Spectropho- 


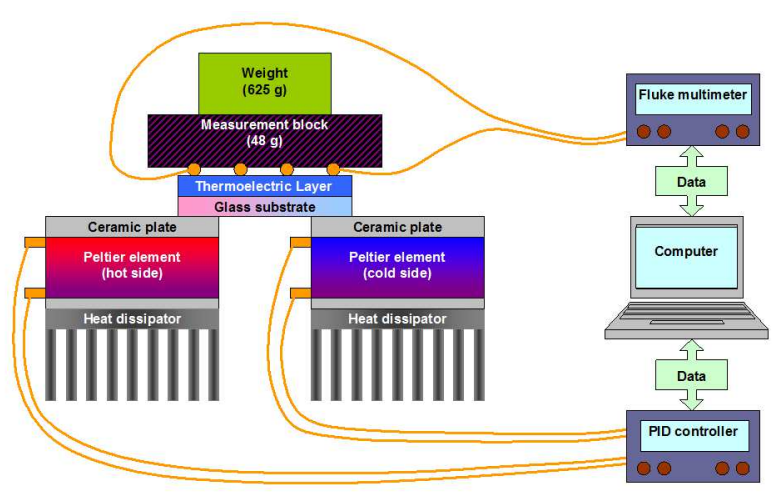

Figure 1: Depiction of the instrument constructed for the measurement of the Seebeck coefficient, in case of the thin samples.

tometer to obtain information on the optical behavior of the thin layers, while Raman spectroscopy reveals detailed insights into the structural changes within the chains. The Raman spectra are obtained using a He-Ne laser with an excitation wavelength of $633 \mathrm{~nm}$, a power of $8 \mathrm{~mW}$ and an accumulation time of $10 \mathrm{~s}$. The excitation light is focused on the solid samples through a microscope objective. The recorded Raman spectra are frequency calibrated using a 50:50 vol. acetonitrile to toluene mixture. The background corrected Raman spectra of all samples are presented in Fig. S9.

Smaller samples $\left(\approx 8 \times 8 \mathrm{~mm}^{2}\right)$ are investigated by $\mathrm{X}$-ray photoelectron spectroscopy (XPS) to gain further information about the surface composition of the samples. XPS spectra are recorded at room temperature, using non-monochromatized $\mathrm{Al} \mathrm{K \alpha}(1486.6 \mathrm{eV})$ excitation and a hemispherical analyzer (Phoibos 150, SPECS). The binding energy scale is calibrated by the standard $\operatorname{Au} 4 \mathrm{f}(7 / 2)$ and $\mathrm{Cu} 2 \mathrm{p}(3 / 2)$ procedure. The theoretical cross sections from Yeh and Lindau are used to calculate the elemental composition (see Tables S3 and S4, Supporting Information). 42 The XPS spectra are shown after Shirley background subtraction, without normalization.

Further experimental details, discussions and examples of recorded data are given in the Supporting Information.

\section{$3 \quad$ Results and Discussions}

To investigate the influence of the surface morphology without altering the chemical composition of PEDOT:PSS (i.e. without changing the chemical doping levels), the experiments are made using both a pristine 1 wt.\% PEDOT:PSS aqueous solution (noted $\mathrm{PP}$ ) and a similar solution, containing 5 vol.\% DMSO (PP5D). The results are illustrated in Fig. 2 for the various spin-coating speeds and in Fig. 3 for the multiple layers.

As revealed in Fig. 2, the change of the spin-coating velocity (v2) has a small effect on the thermoelectric properties of both types of samples. While the thickness of the films is gradually decreasing from $168 \pm 38 \mathrm{~nm}$ in case of v2 $=1000 \mathrm{RPM}$, to $66 \pm 22 \mathrm{~nm}$ for $\mathrm{v} 2=3000 \mathrm{RPM}$, the electric conductivity is only slightly lowered. Accordingly, the difference between the maximal $\sigma$ value, $0.53 \pm 0.12 \mathrm{~S} \mathrm{~cm}^{-1}$, and the minimal one, $0.39 \pm 0.07 \mathrm{~S} \mathrm{~cm}^{-1}$, amounts to approximately $26 \%$. Similar results are also obtained for the PP5D samples. Even though the change in thickness occurs steeper, from $202 \pm 83 \mathrm{~nm}$ to $60 \pm 17 \mathrm{~nm}$, the difference between the maximal $\left(140 \pm 58 \mathrm{~S} \mathrm{~cm}^{-1}\right)$ and minimal $\left(98 \pm 29 \mathrm{~S} \mathrm{~cm}^{-1}\right) \sigma$ values does not surpass $30 \%$. Since this minimal variation is comparable with the typical experimental uncertainty, $\stackrel{40,43,44}{4}$ the differences in the average values of the power factors are mainly governed by the Seebeck coefficients, which revolve around $11.5 \mu \mathrm{VK}^{-1}$ (see Fig. 2a and 2d). This thermopower is consistent with the one reported by Crispin et al., $\frac{39}{2}$ who also use PEDOT:PSS from Agfa-Gevaert.

A different situation can be observed in Fig. 3, where several polymer layers are repeatedly spincoated onto the same glass substrate. For the PP samples, the thickness of the layers (depicted in Fig. 3b) is constantly rising from $101 \pm 25 \mathrm{~nm}$ for a single PEDOT:PSS layer, to $445 \pm 52 \mathrm{~nm}$ for five layers, as expected. Still, this linear growth in thickness is exceeded by the lowering of the electrical resistance. Accordingly, the electrical conductivity is enhanced by more than 4.5 times, from the value of $0.46 \pm 0.11 \mathrm{~S} \mathrm{~cm}^{-1}$ for a single layer, to $2.10 \pm 0.26 \mathrm{~S} \mathrm{~cm}^{-1}$ for five layers. This result is particularly important, since most reported val- 

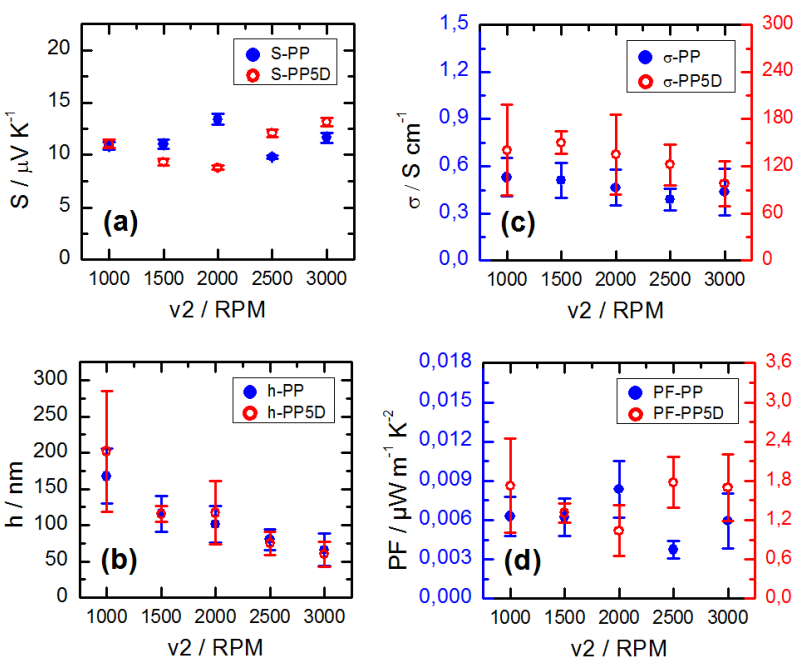

Figure 2: Variation of the thermoelectric properties with the thickness of a single PEDOT:PSS layer (blue - PP solution, red - PP5D solution): (a) Seebeck coefficient, (b) film thickness, (c) electrical conductivity, (d) power factor.
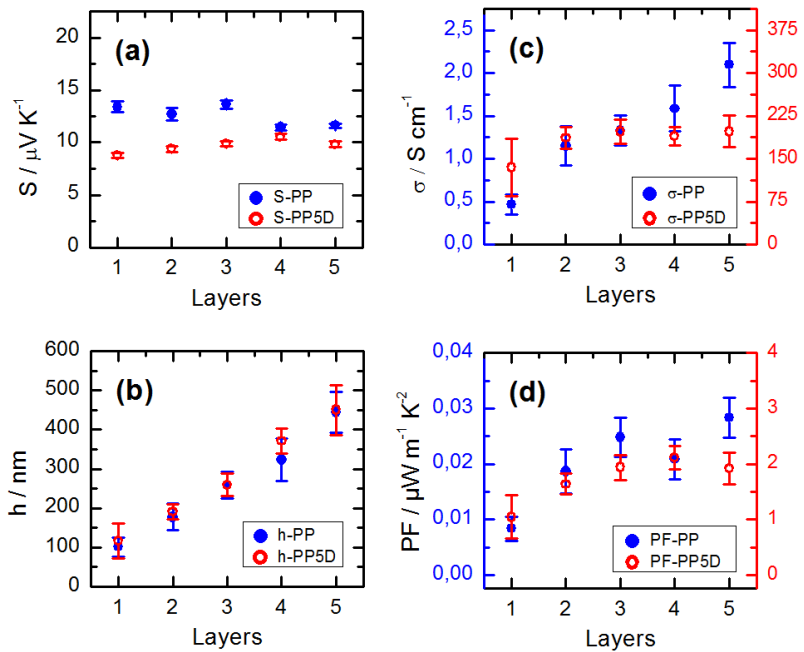

Figure 3: Change in the thermoelectric properties with the number of spin-coated PEDOT:PSS layers (blue - PP solution, red - PP5D solution): (a) Seebeck coefficient, (b) film thickness, (c) electrical conductivity, (d) power factor.
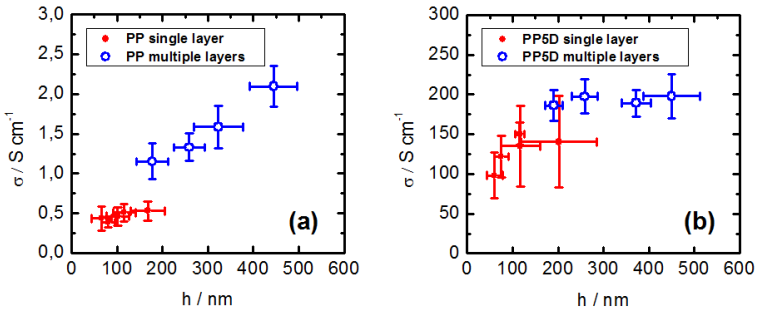

Figure 4: Comparison between the electrical conductivities of the thin films, which are obtained through both the single (red) and the multiple layer approach (blue): (a) PP samples, (b) PP5D samples.

ues amount to less than $1 \mathrm{~S} \mathrm{~cm}^{-1}$ for pristine PEDOT:PSS layers. $\stackrel{439}{ }$ Since the Seebeck coefficient is maintained at approx. $9-13 \mu \mathrm{VK}^{-1}$ (Fig. 31), the power factor remains mostly dependent on the changes in the electrical conductivity, increasing by approximately 3.4 times, from $8.3 \pm 2.2 \mathrm{nW} \mathrm{m}^{-1} \mathrm{~K}^{-2}$ to $28.3 \pm 3.6 \mathrm{nW} \mathrm{m}^{-1} \mathrm{~K}^{-2}$ (Fig. [3 d). Still, the same cannot be said about the PP5D samples, where $\sigma$ appears to reach a plateau at around $200 \mathrm{~S} \mathrm{~cm}^{-1}$, after just three layers (see Fig. 3b), which leads to a maximal power factor of $2.11 \pm 0.22 \mu \mathrm{W} \mathrm{m}^{-1} \mathrm{~K}^{-2}$. In contrast to the $\mathrm{PP}$ samples, the $\mathrm{PF}$ is only enhanced by 1.9 times with respect to the single layer. Nevertheless, this value surpasses the enhancements obtained using the common surface treatment approach (see Section 9, Supporting Information), indicating that a further effect is responsible for the improvement in conductivity.

The results also emphasize the necessity to compare different strategies of thermoelectric enhancement using similar solutions and procedures, since even the type of product or vendor can have a significant influence on the obtained performance. For example, the Seebeck coefficients revolve around 7$25 \mu \mathrm{VK}^{-1}$ for the Heraeus Clevios $\mathrm{P}$ product series (having undisclosed preparation procedures), while the electrical conductivities span between 50$1000 \mathrm{~S} \mathrm{~cm}^{-1}$, resulting in power factors of around 1$50 \mu \mathrm{W} \mathrm{m}^{-1} \mathrm{~K}^{-2}, 2,25,26,30,31,35,36,45$

The connection between the electrical conductivity, the film thickness and the sample preparation is em- 
PEDOT:

(a)

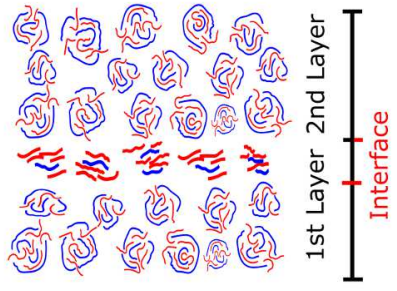

(b)

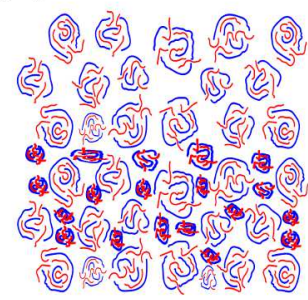

(e)

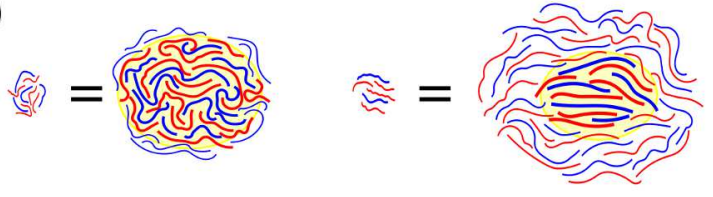

Figure 5: (a-d) Possible mechanisms for enhancing the electrical transport in multi-layered films. The washing and reorganization of the PEDOT:PSS grains can create highly conductive inner surfaces: (a) PP solution, (c) PP5D solution. An inclusion of smaller PEDOT:PSS grains into the existing porous layer can also enhance the percolation between particles, by increasing the amount of contacts between them: (b) PP solution, (d) PP5D solution. (e) Model of the pristine (left) and reorganized (right) PEDOT:PSS grains, where the yellow region corresponds to the crystalline domains in Ref. 46. phasized in Fig. 4, allowing a correlation between the electrical transport within the samples and their morphology. There, both the general increase in $\sigma$ with $h$ and the leap in the electrical conductivity from single to multiple layers are visible. As a first pristine PEDOT:PSS layer is spin-coated, its granular structure leads to a type of transport by percolation, which is largely oriented along the two dimensional template of the glass substrate. Since the self organization of the PEDOT:PSS grains always takes place in a similar matter, no significant difference is observed for the single layers of various thickness. A similar rationale also applies for the PP5D single layers.

In order to clarify the apparently unusual enhancement for the multiple layers, two plausible mechanisms are proposed and depicted in Fig. 5 for both types of solutions (PP and PP5D), namely the washing/reorganization of PSS chains, or the inclusion of smaller polymer grains into the existing porous structure. In the first case (Fig. 5 a), the (partial) solvation of the polar PSS chains (colored blue) in water can lead to inner surfaces with a superior $\sigma$ (see the highlighted chains at the interfaces), enhancing the overall conductivity of the thin film. In the second case (Fig. 5b), the inclusion of smaller particles or even single chains leads to a higher amount of electrical contacts between all grains (i.e. a higher probability that percolation occurs), corresponding to a higher $\sigma$. As the polarity of DMSO accounts for a reorganization of the PEDOT:PSS grains, more packed polymer films are obtained from the PP5D solution, with a better electrical contact between the PEDOT chains. As such, effects like surface washing or reorganization should not play a major role anymore, since they happen intrinsically during the spin-coating of all layers (see Fig. 55). This indicates the inclusion of smaller particles (or even single polymer chains) as the main mechanism for the enhancement of $\sigma$, which is illustrated in Fig. 5b and 5 $\mathrm{d}$. Indeed, since the density of the obtained films cannot be significantly further increased, the electrical conductivity also reaches a plateau for the PP5D samples.

An inclusion is also supported by the AFM images of both PP and PP5D single layers (Fig. 6), which reveal irregular surface profiles, resembling those illustrated in Fig. 5. The two films are imaged at both 

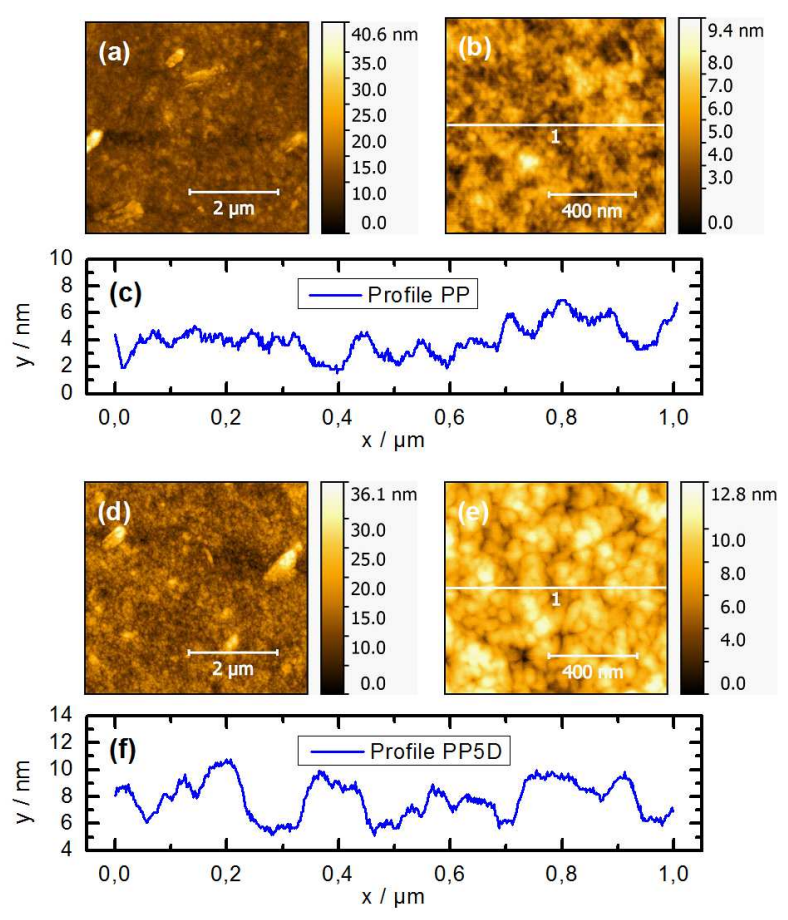

Figure 6: AFM images (a,b,d,e) and surface profiles $(\mathrm{c}, \mathrm{f})$ along: (a-c) the PP samples, (d-f) the PP5D layers. The two profiles correspond to the while lines from frames (b) and (e).

$5 \times 5 \mu \mathrm{m}^{2}$ (6a and 6 $\left.\mathrm{d}\right)$ and $1 \times 1 \mu \mathrm{m}^{2}$ (6b, 6b) resolution, which gives a better representation of the surface morphology. The profiles in Fig. 6r and 6f correspond to the white lines in Fig. $6 \mathrm{~b}$ and 6 , giving a better representation for these surface irregularities. The quantitative root mean squares (RMS) are also determined for the two $1 \times 1 \mu \mathrm{m}^{2}$ AFM images (6 $\mathrm{b}$ and (6e), amounting to $1.17 \mathrm{~nm}$ for the PP surface, respectively $1.63 \mathrm{~nm}$ for the PP5D one. Accordingly, a certain granularity is also maintained for the samples made from the DMSO containing solution, indicating that percolation plays a role in the electrical conduction even in this case. This granularity is consistent with that observed in other reports, although the PEDOT:PSS grain size is often variable (see for example Refs. 32 and 29), resulting again in different electrical conductivities. It is worth mentioning here that the grain size depends not only on the synthetic process, but also on the temperature of the resulting solution. 47.48

These findings also contribute to a better understanding of the macromolecular ordering within conductive polymer films, which is currently still under debate. $3,5,46,48-51$ While some models assume long conjugated chains, $\underline{\underline{50,51}}$ which can aggregate into highly ordered, crystalline domains, $\underline{46,50}$ other models suggest bundled structures, where short PEDOT chains are grouped around longer PSS ones. $\frac{5}{5}$ Similarly, recent depictions of the solvent-driven chain reorganization include either smooth surfaces of densely packed polymer chains, $\underline{\underline{3}}$ or crystalline PEDOT domains within a PSS amorphous matrix. $\underline{46}$ While AFM investigations indicate a granular morphology instead, the microfocused grazing incidence small- and wide-angle X-ray scattering ( $\mu$-GISAXS and $\mu$-GIWAXS) investigations of Palumbiny et al. give further unique insights over the distribution of the crystalline domains and the molecular reorientation within the films. In particular, smaller, more ordered crystalline domains appear to form when using a cosolvent, $\underline{46}$ in contrast with the larger PEDOT:PSS grains observed from AFM images. These opposing effects indicate that the cosolvent addition might simultaneously cause a better crystallinity on the inner PEDOT rich region and a partially amorphous reorganization of the polymer chains on the outer region. In the presence of DMSO as cosolvent, the amorphous PSS shells would grow by including PEDOT chains from the inner region, explaining the enhancement in conductivity. This rearrangement is depicted in Fig. 55, where the yellow highlighted area corresponds to the $\mu$-GISAXS detected crystalline domains from Ref. 46. Our model also takes into account a possible alternating structure of PEDOT and PSS layers, which would be required to avoid Coulomb repulsions between the positive face-on or edge-on $\underline{46}$ oriented PEDOT chains.

Although the previous results support the percolative mechanism, a certain washing or reorganization of the polymer grains at the layer-layer interface is still plausible, as indicated by Raman measurements. There, the correlation between the thickness of the 
films and the intensity of the signal (given in counts per second, or cps) is also visible (Fig. S9, Supporting Information). A close-up of the Raman bands is given in Fig. 7b for the PP multiple layers, where the signal noise is smoothed using a 4 point fast Fourier transform (FFT) filter. The bands at $989 \mathrm{~cm}^{-1}$, $1103 \mathrm{~cm}^{-1}, 1256 \mathrm{~cm}^{-1}$ and $1371 \mathrm{~cm}^{-1}$ can be attributed to the oxyethylene ring deformation, PSS component deformation, $\mathrm{C}_{\alpha}-\mathrm{C}_{\alpha}$ inter-ring stretching, respectively $\mathrm{C}_{\beta}-\mathrm{C}_{\beta}$ stretching. ${ }^{45}$ The most intensive band at around $1400-1450 \mathrm{~cm}^{-1}$, corresponds to the $\mathrm{C}_{\alpha}=\mathrm{C}_{\beta}$ symmetric stretching vibration of the fiveatom ring. 26,45,52,53 For most pristine PEDOT:PSS layers (Fig. S9a and S9b, Supporting Information), the band presents a maximum at $1429 \mathrm{~cm}^{-1}$, corresponding to the presence of both benzenoid and quinoid resonant structures. 26,53 Still, the band is slowly red-shifting towards $1417 \mathrm{~cm}^{-1}, \underline{26,53}$ as the number of layers is increasing. Accordingly, the maximum reaches $1422 \mathrm{~cm}^{-1}$ for the five-layered PEDOT:PSS film. This indicates a transition towards the more linear quinoid structure, which translates in a higher electrical conductivity. 26,53 Although this increase in $\sigma$ has been already determined by electrical measurements, the accompanying structural change is still remarkable, since it occurs without any special treatment. Accordingly, this finding supports an electrical transport through the mechanism presented in Fig. 57. A similar red-shifting is also observed for the PP5D layers (Fig. S10, Supporting Information), indicating that both enhancements have a similar cause. Still, since the PEDOT:PSS chains in the PP5D samples are already mostly reorganized, the lower improvement in the electrical conductivity can be understood.

The UV-Vis absorbance of the layers depends on their thickness, increasing gradually with the number of layers (Fig. 7a and S8d), respectively for a lower spin-coating speed (Fig S8a and S8c, Supporting Information). Overall, the broad near infrared (NIR) absorption indicates the presence of $\mathrm{PEDOT}^{2+}$ bipolarons in the layers from both types of solutions, PP and PP5D (literature values for the UV-Vis absorption bands are given in Table S2 from the Supporting Information). This qualitatively reveals that the PEDOT and PSS chains are only reorganized, without changing their proportion in the mixture.
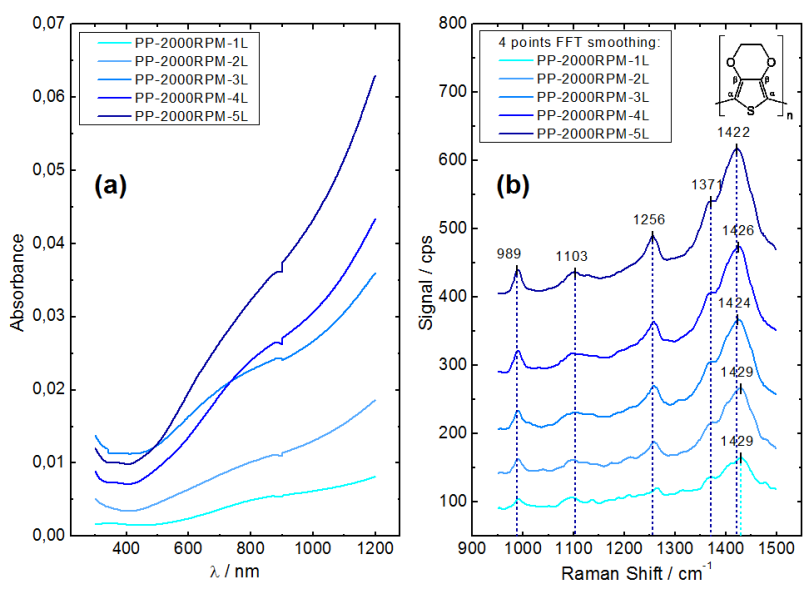

Figure 7: Spectra of the multi-layered PP films: (a) UV-Vis, (b) Raman. The maxima in the Raman bands are determined after smoothing the signal noise with a 4 point fast Fourier transform (FFT) filter (see the Supporting Information for the original full scale spectra). The Raman spectra are shifted vertically by an arbitrary value, in order to avoid overlapping and to improve visibility. The color of both UV-Vis and Raman spectra indicates the layer's thickness, by using darker blue tones for the thicker films. The inset in (b) reveals the structure and notation of neutral PEDOT.

These findings are also supported by quantitative XPS measurements. The relative signal intensity of the $\mathrm{S}(2 \mathrm{p})$ thiophene sulfur peak in PEDOT (at around $167.8 \mathrm{eV}$, see Fig. S12, Supporting Information) and the sulfur $\mathrm{S}(2 \mathrm{p})$ peak originating from PSS (at $164.1 \mathrm{eV}$ ) is maintained at approximately 0.35 for both PP (Fig. 83) and PP5D (Fig. S11, Supporting Information) layers, which indicates that no significant change in the composition of the films occurs at their upper surface (i.e. no washing). Accordingly, the cause for the enhancement lays within the thin film, supporting the mechanisms predicted in Fig. 5. Moreover, XPS spectra also confirm that the addition of DMSO to the aqueous PP solution only reorganizes the chains, without altering the PEDOT to PSS ra- 

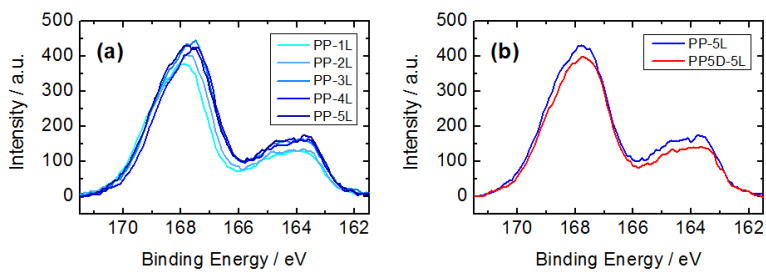

Figure 8: (a) $\mathrm{S}(2 \mathrm{p})$ core level XPS spectra of the PP multiple layers, after Shirley background subtraction. (b) Comparison between the XPS spectra of five-layered films, obtained using either PP or PP5D solutions.

tio. This is exemplified in Fig. 8b by comparing both PP and PP5D five-layered films.

This effect explains the reported literature data, which suggests outstanding electrical conductivities for multiple layers, $\frac{5}{\underline{5}}$ such as the approx. $1900 \mathrm{~S} \mathrm{~cm}^{-1}$ for PEDOT:PSS thin films ${ }^{26}$ or $1885 \mathrm{~S} \mathrm{~cm}^{-1}$ for complex composite multilayers. $\underline{\underline{23}}$ The small variation in the Seebeck coefficient of PEDOT:PSS is also consistent with the findings of several groups, $\underline{26,39}$ which indicates that the enhancement in thermopower reported by Grunlan et al. $\stackrel{23}{ }$ for PANI/graphenePEDOT:PSS/PANI/DWNT-PEDOT:PSS quadlayers is related to the interactions between the various components.

\section{Conclusions}

In conclusion, the thermoelectric properties of $\mathrm{PE}$ DOT:PSS layers can be significantly varied, depending on the spin-coating method. While the electrical conductivity of single layers is approximately constant regardless of their thickness, the $\sigma$ values can be significantly enhanced by spin-coating several layers onto the same substrate. In particular, the electrical conductivity of pristine PEDOT:PSS amounts to more than $2 \mathrm{~S} \mathrm{~cm}^{-1}$ after only 5 layers, surpassing most reported values. A similar effect is also obtained for DMSO-containing PEDOT:PSS solutions. As indicated by AFM, UV-Vis, Raman and XPS measurements, this remarkable enhancement can be attributed to a combination of factors at two different scales: percolation between the grains and reorganization of the conjugated chains.

The multi-layered approach also stands out from the commercial point of view, since it might deliver a direct way of improving the efficiency of printed thin film devices, without needing additional equipment or facilities. Moreover, this technique can offer a better control over the thickness of the resulting films, which can grow above the threshold of a few hundred nanometers. Beyond the potential implications for practical applications, these results contribute to a deeper understanding of the conduction mechanisms in organic polymers, paving the way towards a clarification of the differences between existing reports.

\section{Acknowledgements}

V.A. performed the TE and AFM measurements. K.B. designed and constructed the TE measurement setup. V.A. and F.M. recorded the UV-Vis and Raman spectra. S.B. and A.K.L. recorded the XPS spectra. V.A., K.B., F.M., S.B., A.K.L., J.K. and K.R. were involved in the creation of the manuscript. We are grateful to DFG for the support through the SPP 1415 Programme. V.A. is most grateful to the Gesellschaft Deutscher Chemiker for the AugustWilhelm-von-Hofmann-Scholarship and to the German Federal Government and the Bayer Foundations for the "Deutschlandstipendium" scholarship. K.B. is most grateful for the scholarship from the International Max-Planck Research School at the Fritz Haber Institute.

Keywords: thermoelectrics; conducting polymers PEDOT:PSS; spin-coating; thin films.

\section{References}

[1] A. J. Heeger, J. Phys. Chem. B 2001, 105, 8475-8491.

[2] Q. Zhang, Y. Sun, W. Xu, D. Zhu, Adv. Mater. 2014, 26, 6829-6851.

[3] D. Alemu, H.-Y. Wei, K.-C. Ho, C.-W. Chu, Energy Environ. Sci. 2012, 5, 9662-9671. 
[4] H. Shi, C. Liu, Q. Jiang, J. Xu, Adv. Electron. Mater. 2015, 1, 1500017.

[5] C. M. Palumbiny, J. Schlipf, A. Hexemer, C. Wang, P. Müller-Buschbaum, Adv. Electron. Mater. 2016, 2, 1500377.

[6] Y. Xuan, X. Liu, S. Desbief, P. Leclère, M. Fahlman, R. Lazzaroni, M. Berggren, J. Cornil, D. Emin, X. Crispin, Phys. Rev. B 2010, 82, 115454.

[7] B. Endrôdi, J. Mellár, Z. Gingl, C. Visy, C. Janáky, J. Phys. Chem. C 2015, 119, 84728479 .

[8] Y. Cao, J. Qiu, P. Smith, Synth. Met. 1995, 69, 187-190.

[9] Y. Zhu, J. Zhang, Y. Zheng, Z. Huang, L. Feng, L. Jiang, Adv. Funct. Mater. 2006, 16, 568-574.

[10] A. M. Glaudell, J. E. Cochran, S. N. Patel, M. L. Chabinyc, Adv. Energy Mater. 2015, 5, 1401072-n/a.

[11] M. Culebras, C. M. Gómez, A. Cantarero, Materials 2014, 7, 6701.

[12] V. Andrei, K. Bethke, K. Rademann, Energy Environ. Sci. 2016, 9, 1528-1532.

[13] B. T. McGrail, A. Sehirlioglu, E. Pentzer, Angew. Chem. Int. Ed. 2015, 54, 1710-1723.

[14] C. Yu, Y. S. Kim, D. Kim, J. C. Grunlan, Nano Lett. 2008, 8, 4428-4432.

[15] M. Culebras, C. M. Gómez, A. Cantarero, J. Mater. Sci. 2013, 48, 2855-2860.

[16] B. Dörling, J. D. Ryan, J. D. Craddock, A. Sorrentino, A. E. Basaty, A. Gomez, M. Garriga, E. Pereiro, J. E. Anthony, M. C. Weisenberger, A. R. Goñi, C. Müller, M. Campoy-Quiles, Adv. Mater. 2016, 28, 2782-2789.

[17] S. Walia, S. Balendhran, H. Nili, S. Zhuiykov, G. Rosengarten, Q. H. Wang, M. Bhaskaran, S. Sriram, M. S. Strano, K. Kalantar-zadeh, Prog. Mater. Sci. 2013, 58, 1443-1489.
[18] K. Koumoto, Y. Wang, R. Zhang, A. Kosuga, R. Funahashi, Annu. Rev. Mater. Res. 2010, 40, 363-394.

[19] J. Loureiro, N. Neves, R. Barros, T. Mateus, R. Santos, S. Filonovich, S. Reparaz, C. M. Sotomayor-Torres, F. Wyczisk, L. Divay, R. Martins, I. Ferreira, J. Mater. Chem. A 2014, 2, 6649-6655.

[20] V. Andrei, K. Bethke, K. Rademann, Phys. Chem. Chem. Phys. 2016, 18, 10700-10707.

[21] V. Andrei, K. Bethke, K. Rademann, Appl. Phys. Lett. 2014, 105, 233902.

[22] C. Cho, B. Stevens, J.-H. Hsu, R. Bureau, D. A. Hagen, O. Regev, C. Yu, J. C. Grunlan, Adv. Mater. 2015, 27, 2996-3001.

[23] C. Cho, K. L. Wallace, P. Tzeng, J.-H. Hsu, C. Yu, J. C. Grunlan, Adv. Energy Mater. 2016, 6,1502168 .

[24] Y. Xia, J. Ouyang, J. Mater. Chem. 2011, 21, 4927-4936.

[25] F. Greco, A. Zucca, S. Taccola, A. Menciassi, T. Fujie, H. Haniuda, S. Takeoka, P. Dario, V. Mattoli, Soft Matter 2011, 7, 10642-10650.

[26] D. A. Mengistie, C.-H. Chen, K. M. Boopathi, F. W. Pranoto, L.-J. Li, C.-W. Chu, ACS Appl. Mater. Interfaces 2015, 7, 94-100.

[27] Q. Wang, M. Eslamian, Ultrasonics 2016, 67, 55-64.

[28] B. J. Worfolk, S. C. Andrews, S. Park, J. Reinspach, N. Liu, M. F. Toney, Mannsfeld, Stefan C. B., Z. Bao, Proc. Natl. Acad. Sci. U. S. A. 2015, 112, 14138-14143.

[29] Z. Yu, Y. Xia, D. Du, J. Ouyang, ACS Appl. Mater. Interfaces 2016, 8, 11629-11638.

[30] S. Liu, H. Deng, Y. Zhao, S. Ren, Q. Fu, RSC Adv. 2015, 5, 1910-1917. 
[31] J. Kim, J. G. Jang, J.-I. Hong, S. H. Kim, J. Kwak, J. Mater. Sci.: Mater. Electron. 2016, $1-6$.

[32] J. Ouyang, ACS Appl. Mater. Interfaces 2013, 5, 13082-13088.

[33] N. Kim, H. Kang, J.-H. Lee, S. Kee, S. H. Lee, K. Lee, Adv. Mater. 2015, 27, 2317-2323.

[34] B. Fan, X. Mei, J. Ouyang, Macromolecules 2008, 41, 5971-5973.

[35] O. Bubnova, M. Berggren, X. Crispin, J. Am. Chem. Soc. 2012, 134, 16456-16459.

[36] N. Massonnet, A. Carella, O. Jaudouin, P. Rannou, G. Laval, C. Celle, J.-P. Simonato, J. Mater. Chem. C 2014, 2, 1278-1283.

[37] J. L. Bredas, G. B. Street, Acc. Chem. Res. 1985, 18, 309-315.

[38] O. Bubnova, X. Crispin, Energy Environ. Sci. 2012, 5, 9345-9362.

[39] O. Bubnova, Z. U. Khan, H. Wang, S. Braun, D. R. Evans, M. Fabretto, P. Hojati-Talemi, D. Dagnelund, J.-B. Arlin, Y. H. Geerts, S. Desbief, D. W. Breiby, J. W. Andreasen, R. Lazzaroni, W. M. Chen, I. Zozoulenko, M. Fahlman, P. J. Murphy, M. Berggren, X. Crispin, Nat. Mater. 2014, 13, 190-194.

[40] G. J. Snyder, E. S. Toberer, Nat. Mater. 2008, 7, 105-114.

[41] K. Bethke, V. Andrei, K. Rademann, PLoS ONE 2016, 11, e0151708.

[42] J. J. Yeh, I. Lindau, At. Data Nucl. Data Tables 1985, 32, 1-155.

[43] E. Alleno, D. Bérardan, C. Byl, C. Candolfi, R. Daou, R. Decourt, E. Guilmeau, S. Hébert, J. Hejtmanek, B. Lenoir, P. Masschelein, V. Ohorodnichuk, M. Pollet, S. Populoh, D. Ravot, O. Rouleau, M. Soulier, Rev. Sci. Instrum. 2015, 86, 011301.
[44] K. A. Borup, J. d. Boor, H. Wang, F. Drymiotis, F. Gascoin, X. Shi, L. Chen, M. I. Fedorov, E. Muller, B. B. Iversen, G. J. Snyder, Energy Environ. Sci. 2015, 8, 423-435.

[45] Z. Zhu, C. Liu, Q. Jiang, H. Shi, F. Jiang, J. Xu, J. Xiong, E. Liu, J. Mater. Sci.: Mater. Electron. 2015, 26, 8515-8521.

[46] C. M. Palumbiny, C. Heller, C. J. Schaffer, V. Körstgens, G. Santoro, S. V. Roth, P. MüllerBuschbaum, J. Phys. Chem. C 2014, 118, 13598-13606.

[47] B. Friedel, T. J. Brenner, C. R. McNeill, U. Steiner, N. C. Greenham, Org. Electron. 2011, 12, 1736-1745.

[48] J. Zhou, D. H. Anjum, L. Chen, X. Xu, I. A. Ventura, L. Jiang, G. Lubineau, J. Mater. Chem. C 2014, 2, 9903-9910.

[49] A. A. Paraecattil, N. Banerji, J. Am. Chem. Soc. 2014, 136, 1472-1482.

[50] S. D. Kang, G. J. Snyder, Nat. Mater. 2016, advance online publication. DOI: $10.1038 /$ nmat4784.

[51] J. J. Urban, Nat. Mater. 2016, advance online publication. DOI: $10.1038 /$ nmat4790.

[52] J. Ouyang, C.-W. Chu, F.-C. Chen, Q. Xu, Y. Yang, Adv. Funct. Mater. 2005, 15, 203-208.

[53] J. E. McCarthy, C. A. Hanley, L. J. Brennan, V. G. Lambertini, Y. K. Gun'ko, J. Mater. Chem. C 2014, 2, 764-770. 